\title{
Ultrasound guidance versus direct palpation for radial artery catheterization by expert operators: a randomized trial among Canadian cardiac anesthesiologists Échoguidage versus palpation directe pour la canulation de
l'artère radiale par des spécialistes: une étude randomisée auprès
d'anesthésiologistes cardiaques canadiens
}

\author{
Cheryl Peters, MD · Stephan K. W. Schwarz, MD, PhD - Cynthia H. Yarnold, MD •
} Katarina Kojic $\cdot$ Stefan Kojic, BSc $($ Pharm) $\cdot$ Stephen J. Head, MD

Received: 16 February 2015/Revised: 4 May 2015/Accepted: 23 June 2015/Published online: 10 July 2015

(c) Canadian Anesthesiologists' Society 2015

\begin{abstract}
Purpose The use of ultrasound (US) for radial arterial catheterization has been reported to result in faster insertion times with fewer complications when compared with traditional direct palpation $(D P)$. We sought to determine if this applies to expert operators and tested the hypothesis that, among cardiac anesthesiologists, USguided insertion similarly results in faster insertion times as well as fewer re-directs, attempts, and complications.
\end{abstract}

A preliminary account of this work has been presented in abstract form at ISURA $2014\left(11^{\text {th }}\right.$ Annual International Symposium of Ultrasound for Regional Anesthesia, Pain Medicine, and Perioperative Application); Toronto, ON; June 21-24, 2014.

Author contributions Cheryl Peters, Katarina Kojic, and Stefan Kojic were involved in patient recruitment and data acquisition. Cheryl Peters wrote the first draft of the manuscript. Stephan K. W. Schwarz and Cynthia H. Yarnold were involved in the conception and design of the study. Stephan K. W. Schwarz performed the data analysis and critically edited the manuscript. Stephen J. Head conceived the study, was involved in design, manuscript writing, and editing, and reviewed the original study data.

C. Peters, MD - S. K. W. Schwarz, MD, PhD ( $₫)$.

C. H. Yarnold, MD · K. Kojic · S. Kojic, BSc (Pharm) .

S. J. Head, MD

Department of Anesthesia, St. Paul's Hospital, Providence Level

3, 1081 Burrard Street, Vancouver, BC V6Z 1Y6, Canada

C. Peters, MD - S. K. W. Schwarz, MD, PhD .

C. H. Yarnold, MD · K. Kojic · S. Kojic, BSc (Pharm) .

S. J. Head, MD

Department of Anesthesiology, Pharmacology \& Therapeutics,

The University of British Columbia, Vancouver, BC, Canada
Methods Following Research Ethics Board Approval, we conducted a randomized clinical trial in 125 patients scheduled for cardiac surgery at a single tertiary/ quaternary care centre. Patients were randomized to either US- or DP-guided radial artery catheterization by attending cardiac anesthesiologists. The primary endpoint was time to catheter placement. Secondary endpoints included the number of attempts and re-directs as well as the failure rate.

Results There were no differences between the DP- $(n=$ 62) vs US-guided $(n=63)$ groups in median [interquartile range] time to placement (104 [76-212] sec vs 104 [68$270]$ sec, respectively; $P=0.66)$, number of re-directs $(2$ [0-6] vs 3 [1-5], respectively; $P=0.82)$, or number of attempts (1 [1-2] vs 1 [1-2], respectively; $P=0.08)$. The first-attempt success rate was $56.4 \%$ in the DP group and $71.4 \%$ in the US group $(P=0.10)$. Failure rate and hematoma rate in the DP group were $21.0 \%$ and $22.6 \%$, respectively, compared with $12.7 \%$ and $11.1 \%$ in the US group $(P=0.24$ and 0.10 , respectively).

Conclusions Among experienced cardiac anesthesiologists, the use of US to facilitate radial arterial catheterization did not affect insertion times, the number of re-directs, or the number of attempts when compared with DP. Ultrasound use had no significant effects on the rates of success on first attempt, failure, or hematoma formation. This trial was registered at www.clinicaltrials. gov: NCT02118441.

Résumé

Objectif Selon les études, l'utilisation de l'échoguidage (EG) pour la canulation de l'artère radiale réduit les temps 
d'insertion et le nombre de complications par rapport à la palpation directe (PD), qui est la méthode traditionnelle. Notre objectif était de savoir si cette observation s'appliquait aux spécialistes et avons testé l'hypothèse suivante: est-ce que, lorsqu'elle est réalisée par des anesthésiologistes cardiaques, l'insertion avec échoguidage entraîne également une réduction du temps d'insertion, du nombre de tentatives, de changements de direction de la sonde, ou du nombre de complications?

Méthode Après avoir obtenu le consentement du Comité d'éthique de la recherche, nous avons réalisé une étude clinique randomisée auprès de 125 patients devant subir une chirurgie cardiaque dans un seul centre de soins tertiaires / quaternaires. Les patients ont été randomisés à subir une canulation de l'artère radiale par EG ou par PD, réalisée par les anesthésiologistes cardiaques de l'institution. Le critère d'évaluation principal était le temps nécessaire au bon positionnement du cathéter. Les critères d'évaluation secondaires comprenaient le nombre de tentatives d'insertion, de changements de direction de la sonde, ainsi que le taux d'échec.

Résultats Aucune différence n'a été observée entre les groupes PD $(n=62)$ et EG $(n=63)$ en matière de temps médian [écart interquartile] jusqu'au positionnement (104 [76-212] sec vs 104 [68-270] sec, respectivement; $P=$ 0,66), du nombre de changements de direction de la sonde (2 [0-6] vs 3 [1-5], respectivement; $P=0,82$ ), ou $d u$ nombre de tentatives (1 [1-2] vs 1 [1-2], respectivement; $P=0,08)$. Le taux de réussite à la première tentative était de 56,4\% dans le groupe PD et de 71,4\% dans le groupe EG $(P=0,10)$. Les taux d'échec et d'hématome dans le groupe PD étaient de $21,0 \%$ et $22,6 \%$, respectivement $(P$ $=0,24)$, par rapport à $12,7 \%$ et $11,1 \%$, respectivement $(P$ = 0,10), dans le groupe EG.

Conclusion Lorsque pratiqué par des anesthésiologistes cardiaques d'expérience, le recours à l'échoguidage pour la canulation de l'artère radiale n'a pas eu d'impact sur le temps d'insertion, ni sur le nombre de tentatives ou de changements de direction de la sonde par rapport à la palpation directe. L'échoguidage n'a pas non plus eu d'effet significatif sur le taux de réussite à la première tentative, d'échec ou de formation d'hématomes. Cette étude a été enregistrée au www.clinicaltrials.gov: NCT02118441.

Radial artery cannulation is a common procedure in the care of patients in the perioperative period. Accurate continuous "beat-to-beat" hemodynamic monitoring and repeated blood sampling are frequent indications. As a perioperative procedure performed by anesthesiologists, radial catheter insertion is one of a number of tasks that may contribute to anesthesia-controlled time (ACT; defined as the time starting from when the patient enters an operating room to when positioning or skin preparation can begin plus the time starting from when the surgical dressing is completed to when the patient leaves the operating room). ${ }^{1}$ In 1990 , approximately 8 million radial arterial catheters were inserted in the United States. ${ }^{2}$ As the perioperative care of our aging population becomes more complex, these numbers would be expected to increase over the coming decades.

In 1961, Barr was the first to describe radial artery cannulation using a Teflon $^{\circledR}$ catheter $^{3}$; however, from approximately 1955-1970, surgical cutdown was the method of choice. ${ }^{4}$ Since this time, a percutaneous technique using direct palpation (DP) and anatomical landmarks has been favoured.

For perioperative intra-arterial pressure monitoring, the radial artery at the wrist is the site most frequently catheterized as it is easily accessible and has a relatively low complication rate. ${ }^{5}$ The radial artery usually runs a favourably superficial course compared with the deeper and more indirect course of the ulnar artery. ${ }^{4}$ In $30 \%$ of individuals, however, there may be variations in the radial artery's origin and course. ${ }^{6}$ In addition to the insertion challenges posed by anatomic variation, the procedure may be more difficult in patients who are obese, hypotensive, or edematous, which may lead to multiple insertion attempts or failed insertions. ${ }^{7}$ Multiple attempts may produce arterial spasm, which may render further attempts even more challenging and increase patient discomfort.

There has long been substantial evidence that ultrasound (US) guidance for central venous catheter (CVC) insertion, described in $1978,{ }^{8}$ leads to an improved success rate on the first attempt as well as a reduced complication rate. ${ }^{9-11}$ Thus, since 2001, the Agency for Healthcare Research and Quality has recommended that US guidance be used for all CVC insertions. ${ }^{12}$ In contrast, in their 2012 Guidelines, the Councils on Intraoperative Echocardiography and Vascular Ultrasound of the American Society of Echocardiography and the Society of Cardiovascular Anesthesiologists ${ }^{13}$ do not recommend routine real-time US use for arterial cannulation as a general rule. This may partly relate to the fact that comparatively few data exist on the potential advantages of US-guided radial artery insertion. While a number of studies before 2014 have reported an increase in first-attempt success rate, fewer attempts, and a shorter overall time for catheter insertion, ${ }^{5,14,15}$ others found no advantage to using Doppler US, ${ }^{16}$ save for a select group of patients with hemorrhagic shock. ${ }^{17}$ In 2014 , two systematic reviews/meta-analyses of randomized controlled trials dedicated to US-guided radial artery catheterization were published. $^{18,19}$ While both concluded that US use is associated with improved first-attempt success, a small 
underlying evidence base continued, and a majority of the included trials were dedicated to pediatric patients. Until 2014, there were only three included trials on adult patients; two were conducted in an emergency department setting, ${ }^{14,20}$ and one was performed in the operating room setting. The perioperative trial involved a mixed cohort of residents and attending anesthesiologists, many of whom had no prior experience with US-guided radial artery catheterization. ${ }^{21}$ Sample sizes were small, ranging from 60-72; hence, a paucity of evidence continues to exist in the non-pediatric operating room setting on the comparative effects of US-guided radial artery catheterization among expert operators.

Consequently, the purpose of the present randomized controlled trial was to test the hypothesis that US use will result in faster insertion times with fewer complications when performed by experts in palpation-guided radial artery line insertion (i.e., attending Canadian cardiac anesthesiologists) with prior experience in US-guided radial artery catheterization.

\section{Methods}

Following approval by the Providence Health Care Research Ethics Board (Vancouver, BC, Canada; June 7, 2013), we conducted a randomized controlled parallelgroup trial. The study took place in the cardiac operating rooms of an urban academic tertiary/quaternary care centre (St. Paul's Hospital; Vancouver, B.C.) from September 2013 to January 2014. All cardiac anesthesiologists involved in the study had performed a minimum of 300 palpation-guided and 10 US-guided arterial catheter insertions prior to trial commencement. No anesthesia assistants, residents, or other trainees were operators in this study.

Patients were eligible for this study if they were $\geq 18 \mathrm{yr}$ of age, were undergoing cardiac surgery, and had provided written informed consent. Exclusion criteria included a suspected inability to comply with study procedures (including language difficulties or medical history and/or concomitant disease) as judged by the investigator; previous surgery at the site of the proposed radial artery catheterization; any vascular condition that would preclude eligibility for radial artery line insertion as judged by the investigator; patients with a ventricular assist device (no palpable arterial pulsatility); and previous inclusion in this study.

Following enrolment and prior to surgery, patients were randomly allocated to receive one of two techniques of radial artery catheter insertion: the standard DP technique or the US-guided technique. We used consecutively numbered sealed opaque envelopes containing individual folded group assignment cards that were generated (C.P.) prior to commencement of enrolment via urn randomization in blocks of six to minimize selection bias and keep group sizes balanced. After enrolment of an individual patient, one sealed envelope was drawn and opened by the patient's attending cardiac anesthesiologist who performed the allocated study intervention (below). It was not possible to blind data collectors to group allocation because data collection required direct observation of all insertions. The data analyst (S.K.W.S.) was unaware of group allocation until final endpoint variable analyses had been completed and the code was broken.

\section{Study interventions}

Patients were transferred to the cardiac operating room where intravenous access was gained and continuous electrocardiography, pulse oximetry, and noninvasive blood pressure were monitored. Patients were lightly sedated with midazolam $0.5-1 \mathrm{mg} i v$ and/or sufentanil 5$10 \mathrm{ug} i v$ and/or fentanyl $25-50 \mu \mathrm{g}$ at the discretion of the attending anesthesiologist with the goal of maintaining verbal contact with the patient at all times. Patients were positioned supine with their arms slightly abducted and externally rotated at the shoulders. Tape and a rolled towel or intravenous solution bag were used to position and secure the patient's wrist in moderate extension. Antiseptic was applied to the wrist prior to arterial catheter insertion, and local anesthetic in the form of $1 \%$ lidocaine $0.2-1.0 \mathrm{~mL}$ was infiltrated superficially over the target structure at the discretion of the attending anesthesiologist. A standard Arrow $^{\circledR} 20 \mathrm{G} \times 4.45 \mathrm{~cm}$ polyurethane radial artery catheterization set with integral spring-wire guide (RA04020; Arrow International Inc., Reading, PA, USA/ Teleflex Medical, Athlone, Ireland) was used in all cases. Following successful catheterization of the radial artery (below), the arterial catheter was immediately connected to a previously primed pressure transduction set, connected to the monitoring system, and zeroed. Catheters were secured to the skin with a sterile covering (Tegaderm $^{\mathrm{TM}}, 3 \mathrm{M}$, London, ON, Canada), and the patients proceeded to undergo anesthetic care according to routine clinical practice at the discretion of the attending anesthesiologist.

For patients in the DP group, traditional "over-the-wire" radial artery catheter insertion was performed similar to the technique illustrated by Tegtmeyer et al. ${ }^{22}$ Using this approach, operators palpated the radial artery proximal to the intended insertion site for maximum pulsatility near the wrist in the distal lateral forearm. Needles were inserted at an approximate $30-45^{\circ}$ angle relative to the skin, and the integral spring-wire guide of the catheterization set was advanced once a flashback of blood was obtained.

For patients in the US group, a $10-5 \mathrm{MHz} 25-\mathrm{mm}$ linear array US transducer for the SonoSite iLook $^{\circledR}$ system 
(SonoSite, Inc., Bothell, WA, USA) was applied proximal to the intended insertion site near the patient's wrist in the distal lateral forearm in an out-of-plane orientation. Direct palpation was not permitted once the US probe was placed on the patient's wrist. The artery was identified by visible pulsations and lack of compressibility of the vessel (as compared with venous structures). When required, colour flow Doppler was used at the discretion of the operator as an aid in identifying the artery. Needle insertion was performed under "real-time" ultrasonic visualization; needle angulation and wire advancement were executed in a manner similar to the DP group.

\section{Study endpoints and definitions}

The primary endpoint variable was time to successful catheterization. Secondary endpoints included the number of attempts, number of re-directs, first-pass success rate, incidence of hematoma formation, and overall failure rate.

Time zero for the DP group began when the anesthesiologist's fingers were placed on the patient with the purpose of palpating the artery. Time zero for the US group began when the US transducer was first placed on the patient's skin for the purpose of identifying the radial artery. Time to placement was defined as the interval from time zero until the time at which an arterial tracing was viewed on the monitor. An attempt was defined as a new purposeful penetration of the skin with the needle (i.e., following complete withdrawal of the needle from the skin). A re-direct was defined as the needle being purposefully withdrawn at least $5 \mathrm{~mm}$ and re-directed (but not removed from the skin entirely). A failure was declared when the operator was unable to cannulate the radial artery within five minutes. Following a failure, operators were permitted to either persist until successful catheterization was achieved or cross over to the alternative technique.

\section{Statistical analysis}

The projection of the trial's sample size was based on the results of a prior practice audit of 20 DP-guided radial artery catheter placements performed by cardiac anesthesiologists during 2012. The audit yielded a mean (standard deviation) insertion time of 195 (136) sec. We considered time savings of $90 \mathrm{sec}$ to be clinically significant. Based on a target power of $80 \%$ and $\alpha=0.05$ (two-sided), the minimum initial sample size was 36 per group (http://www.stat.ubc.ca/ rollin/stats/ssize/n2.html). To account for the possibility that some patients would be excluded from the analysis for various reasons or the prospect of a non-Gaussian distribution of time-to-event data, we set the initial target sample size to 50 patients per group (total $n=100$ ) for randomization. After randomization of the first eight blocks (24 patients per group), we conducted a blinded interim analysis to assess variances. As a result, we adjusted the target sample size to a minimum of 60 patients per group (total $n=120$ ).

Statistical analysis was conducted on the basis of an "intention-to-treat" approach. In addition, we compared the number of 'failures' (cf., above) between both groups and captured any instances of crossover from the allocated technique to the alternate technique following such declarations of 'failure'. We compared continuous data with the Mann-Whitney test and expressed results as median (interquartile range [IQR]). The difference between medians was assessed by computing the Hodges-Lehmann estimate. We constructed Kaplan-Meyer curves for the primary endpoint and compared the underlying time-toevent data with the log-rank test. For comparisons of categorical data, we used Fisher's exact test to calculate differences between proportions and their $95 \%$ confidence intervals (CIs). All reported $P$ values are two-sided. Prism (GraphPad, San Diego, CA, USA) and Microsoft Excel (Microsoft Corporation, Redmond, WA, USA) software were used for the analyses.

\section{Results}

Over a nine-month period, 129 patients were assessed for eligibility for this study. There were four exclusions based on the stated exclusion criteria. One hundred twenty-five patients were included and randomized, resulting in 63 patients allocated to the US-guided group and 62 patients allocated to the DP-guided group. The trial was ended as the minimum target sample size of 60 per group had been surpassed. All patients were analyzed in the groups to which they were allocated. Fig. 1 provides a study flow chart.

Baseline demographic and surgical data of the patients were similar in both groups, including sex, age, body mass index, American Society of Anesthesiolgists physical status, presence of peripheral vascular disease, systolic blood pressure, and type of surgery (Table 1). Radial arterial catheterizations were performed by 19 cardiac anesthesiologist operators in the DP group and 16 in the US group.

Regarding the primary endpoint, there was no difference between the groups in the median [IQR] time to successful radial artery catheterization (DP, 104 [76-212] sec vs US, 104 [68-270] sec; Hodges-Lehmann estimate for the difference between medians, 6; $P=0.66$ ). Fig. 2 shows scatter plots of the raw primary endpoint data. Fig. 3 illustrates Kaplan-Meyer curves for all patients who were treated. The fastest times to successful catheterization were 


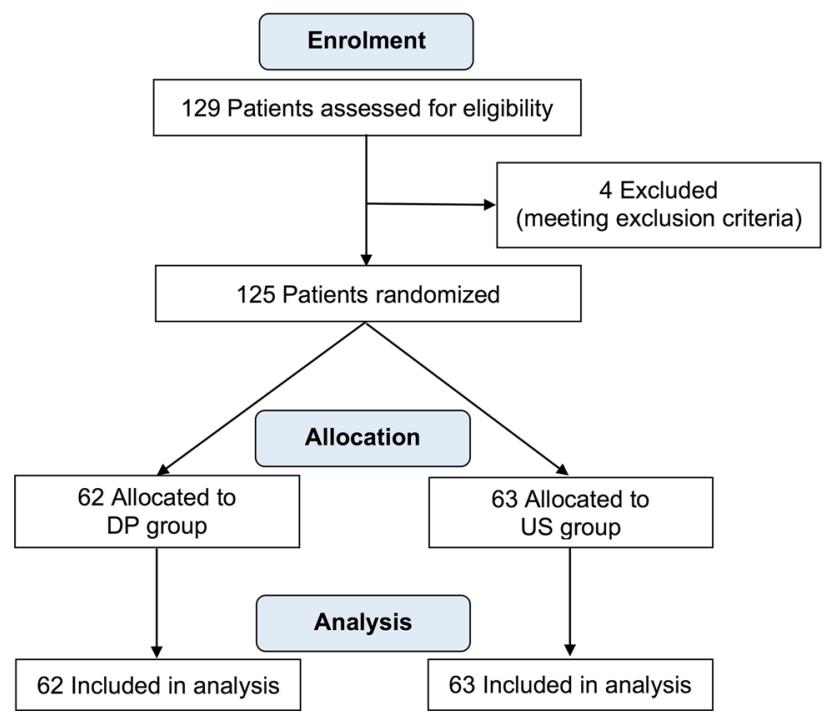

Fig. 1 Study flow chart

Table 1 Patient demographic and surgical data

\begin{tabular}{lcc}
\hline & $\begin{array}{l}\text { Direct palpation } \\
(n=62)\end{array}$ & $\begin{array}{c}\text { Ultrasound } \\
(n=63)\end{array}$ \\
\hline Male sex & $46(74 \%)$ & $52(83 \%)$ \\
Age $(\mathrm{yr})$ & $67(14)$ & $67(14)$ \\
Body mass index $\left(\mathrm{kg} \cdot \mathrm{m}^{-2}\right)$ & $27(5)$ & $26(4)$ \\
ASA physical status IV & $59(95 \%)$ & $36(86 \%)$ \\
Peripheral vascular disease & $3(5 \%)$ & $3(5 \%)$ \\
Systolic blood pressure $(\mathrm{mmHg})$ & $140(18)$ & $140(21)$ \\
Type of surgery & & $33(52 \%)$ \\
CABG & $30(48 \%)$ & $7(11 \%)$ \\
CABG + valve & $4(6 \%)$ & $5(8 \%)$ \\
Valve & $10(16 \%)$ & $14(22 \%)$ \\
TAVI & $12(19 \%)$ & $4(6 \%)$ \\
Lead extraction & $4(6 \%)$ & $0(0 \%)$ \\
LVAD implantation & $1(2 \%)$ & $0(0 \%)$ \\
Pacemaker & $1(2 \%)$ & \\
\hline
\end{tabular}

ASA = American Society of Anesthesiologists; CABG = coronary artery bypass grafting; LVAD = left ventricular assist device; TAVI = transcatheter aortic valve implantation. Age, body mass index, and systolic blood pressure (measured noninvasively at baseline) are given as mean (standard deviation)

observed in the DP group (21 and $30 \mathrm{sec}$ ); the fastest times recorded in the US group were 41 and 49 sec.

Similarly, there was no significant difference between the DP and US groups in the median [IQR] number of attempts (1 [1-2] vs 1 [1-2], respectively; $P=0.08$ ) and number of redirects $(2[0-6]$ vs $3[1-5]$, respectively; $P=0.82)$. Firstattempt success rates were $56.4 \%$ in the DP group and $71.4 \%$ in the US group (difference between proportions, $15.0 \%$; $95 \% \mathrm{CI},-1.9$ to $31.8 ; P=0.10)$. Failure rates were $21.0 \%(n=$ 13 ) and $12.7 \%(n=8)$, respectively (difference between

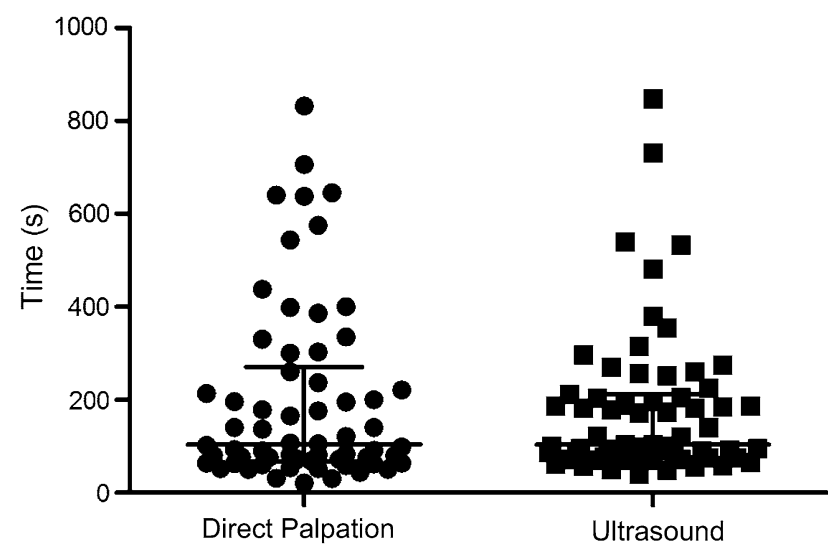

Fig. 2 Primary endpoint variable: time to successful catheterization. Bars represent median and interquartile range

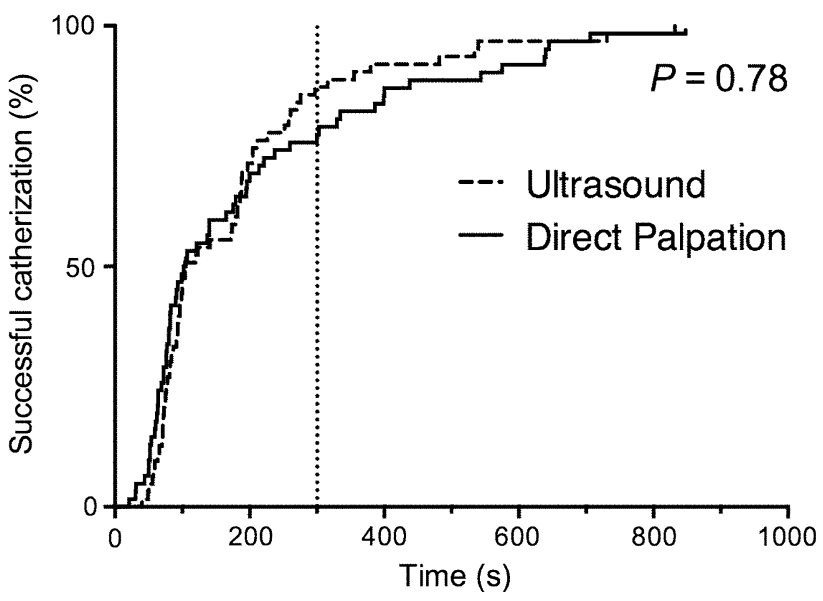

Fig. 3 Kaplan-Meyer curves for the primary endpoint (intention-totreat analysis). The dotted vertical line represents the five-minute mark used to declare a failure (cf. Methods, section Study endpoints and definitions). The $P$ value was calculated with the use of the logrank test; median "survival" was $104 \mathrm{sec}$ in both groups (ratio, 1.00; $95 \%$ confidence interval, 0.70 to 1.41 )

proportions, $8.3 \%$; $95 \% \mathrm{CI}$, -4.8 to $21.4 ; P=0.24$ ). In six of the 13 cases of 'failure' in the DP group, operators used US as rescue after five minutes; in the remaining seven cases, operators persisted with the DP-guided approach. In the US group, operators switched to DP after five minutes in three out of the eight cases of 'failure' and persisted with US use in the other five cases. The rates of hematoma formation in the DP vs US groups were $22.6 \%$ vs $11.1 \%$, respectively (difference between proportions, $11.5 \%$; $95 \% \mathrm{CI},-1.6$ to 24.6; $P=0.10)$.

\section{Discussion}

In the present randomized controlled trial, we found that US use compared with DP had no effect on insertion times 
in adult cardiac surgery patients when performed by expert operators - attending Canadian cardiac anesthesiologists with prior experience in US-guided radial artery catheterization. Likewise, and somewhat similar to the results from an older Japanese study, ${ }^{16}$ we observed that US use had no significant effects on the number of attempts, number of re-directs, first-attempt success rates, failure rates, or hematoma formation, although it should be emphasized that the present trial was not powered to look at these secondary endpoints. On the other hand, an important corollary of these results is that US use neither increased radial artery catheterization times (that might contribute to potential increases in ACT) nor was associated with any other apparent disadvantages.

Our observation that US use did not affect insertion time is consistent with the finding in a recent smaller randomized crossover trial conducted in Denmark by Hansen et al. on a similar cohort of 40 patients. Using an US-guided "dynamic needle tip positioning technique" (a modified short-axis approach), the authors found no difference in the primary study endpoint, needle manipulation time, when compared with DP. ${ }^{23}$ Nevertheless, these authors found that US use increased the first-attempt success rate and decreased the number of attempts. It is important to point out that all US-guided insertions were performed by a single operator, which inherently limits the generalizability of these data. On the other hand, regarding the latter secondary endpoints, Levin et al. similarly reported that US-guidance improved the first-pass success rate (DP, 34\% vs US, 62\%) and decreased the number of attempts, and in this study, no significant difference in insertion times was found. ${ }^{21}$

In contrast, Shiver et al. found a significantly shorter time to successful cannulation, fewer anatomic sites used, and fewer attempts with an US-guided technique compared with a palpation-guided technique. ${ }^{14}$ The disparity of these results when compared with those of our study most likely relates to differences in operator experience. In the former study, the majority of operators were residents; only $13 \%$ were attending anesthesiologists. In the latter study, the operators were emergency medicine physicians. In contrast, all operators in our study were experienced attending cardiac anesthesiologists who routinely place radial artery catheters with or without US, and in this group of operators, US offered no time-saving benefit.

In the pediatric population, mixed results have been obtained with US-guided radial catheter insertion. Schwemmer et al. studied 30 infants and reported an improved success rate as well as fewer attempts in the US group as compared with the DP group. ${ }^{15}$ On the other hand, in another prospective trial involving 152 children by Ganesh et al., US guidance did not facilitate faster cannulation of the radial artery. ${ }^{24}$ A possible explanation for this may again relate to operator inexperience. It was noted in the article that experienced operators (supervising consultant anesthesiologists) were successful where an initial inexperienced operator failed. Consistent with this notion, Ganesh et al. had low first-pass success rates in both the US (13.8\%) and DP (13.9\%) groups.

A potential limitation of any study that attempts to compare two techniques or procedures is not only the overall level of operator experience and seniority but also the potential disparity in operator experience among the two techniques examined. While the present study focused on expert operators (attending cardiac anesthesiologists, all of whom had previously utilized both techniques), individuals' cumulative career experience with the DP technique likely far outweighed that with US-guidance. This disparity, reflected by the minimum required number of US-guided insertions (i.e., ten) prior to study commencement, reflects the "real world" fact that the vast majority of insertions in routine daily practice at our centre have been DP-guided, with US historically used in cases where the DP technique had failed or technical difficulties were expected (e.g., patients with ventricular assist devices). Hence, on the one hand, it is possible that insertion times will decrease with more frequent daily use; on the other hand, all involved cardiac anesthesiologists had routinely been using US for CVC insertion as per standard practice. From this point of view, the finding that US use did not result in increased insertion times suggests to us that this technique indeed represents a useful routine tool even for the most experienced of operators. Nonetheless, areas of further study would be to compare these two techniques among operators who are similarly highly experienced with US-guidance as well as DP techniques and to define the effects of educational interventions that combine hands-on training with web-based e-learning, recently shown to improve proficiency in focused bedside US performance. ${ }^{25}$

Another potential limitation of the present trial relates to the definition of the time interval for the primary endpoint. In our study, 'time zero' in the US group did not occur until the operator placed the US probe on the patient's wrist with the intention of locating the artery. An argument could be made that time zero should have been set at the point in which the US machine is turned on (and before a sterile covering is applied). Nevertheless, in our practice, anesthesia assistants prepare the US machines and probes while the anesthesiologist is completing other tasks, and thus, in our view, the definition used in our study most accurately reflects the "real world" time required for this procedure inasmuch as it affects ACT.

While our primary endpoint variable data robustly show that both groups were very similar (as illustrated by Fig. 2, 
the respective IQRs, and the Hodges-Lehmann estimate for the difference between medians) and our sample size was larger than that in most previous trials dedicated to this topic, we cannot exclude the possibility that studying more patients might have revealed statistically significant differences in such secondary endpoints as first-attempt success rate, failure rate, or hematoma formation. Whereas the present trial was designed and powered with time to successful catheterization in mind and the results do not necessarily imply that there is no benefit to US use on the above secondary endpoints, it nevertheless appears likely that such differences would be relatively small in the context of the present setting.

Finally, our trial focused on acute outcomes and was not intended or designed to investigate more delayed complications such as arterial catheter colonization and associated infection. While Koh et al. reported that the risk of catheter-related bloodstream infection associated with radial arterial catheterization is low compared with central venous catheterization, catheter colonization was in fact similar. ${ }^{26}$ Dedicated future research into the effects of routine US use for radial artery catheterization on these clinically important complications is warranted and will contribute to our knowledge base.

In conclusion, based on the findings of the present trial, we reject our hypothesis that US use will result in faster catheter insertion times with fewer complications when performed by experts in palpation-guided radial artery cannulation - attending Canadian cardiac anesthesiologists. Among this cohort of experienced operators, we did not find that US use significantly improved the rates of success on first attempt, or failure, in an adult cardiac surgery patient population. On the other hand, the results show that US is a useful tool for routine radial artery catheterization in the operating room as its use causes no apparent delay or other significant disadvantages when compared with traditional palpation.

Conflicts of interest None of the authors have any competing financial interests relating to patents and/or shareholdings in corporations involved in the development and/or marketing of any medication or medical device used in this study.

Funding This study was funded by departmental sources (Department of Anesthesia, St. Paul's Hospital, Vancouver, BC). Dr. S. K. W. Schwarz holds the Dr. Jean Templeton Hugill Chair in Anesthesia, supported by the Dr. Jean Templeton Hugill Endowment for Anesthesia Memorial Fund.

\section{References}

1. Dexter F, Coffin S, Tinker JH. Decreases in anesthesia-controlled time cannot permit one additional surgical operation to be reliably scheduled during the workday. Anesth Analg 1995; 81: 1263-8.

2. Gardner RM. Direct arterial pressure monitoring. Curr Anaesth Crit Care 1990; 1: 239-46.

3. Barr PO. Percutaneous puncture of the radial artery with a multipurpose Teflon catheter for indwelling use. Acta Physiol Scand 1961; 51: 343-7.

4. Brzezinski M, Luisetti T, London MJ. Radial artery cannulation: a comprehensive review of recent anatomic and physiologic investigations. Anesth Analg 2009; 109: 1763-81.

5. Scheer B, Perel A, Pfeiffer UJ. Clinical review: complications and risk factors of peripheral arterial catheters used for haemodynamic monitoring in anaesthesia and intensive care medicine. Crit Care 2002; 6: 199-204.

6. McCormack LJ, Cauldwell EW, Anson BJ. Brachial and antebrachial arterial patterns; a study of 750 extremities. Surg Gynecol Obstet 1953; 96: 43-54.

7. Shiloh AL, Savel RH, Paulin LM, Eisen LA. Ultrasound-guided catheterization of the radial artery: a systematic review and metaanalysis of randomized controlled trials. Chest 2011; 139: 524-9.

8. Ullman JI, Stoelting RK. Internal jugular vein location with the ultrasound Doppler blood flow detector. Anesth Analg 1978; 57: 118.

9. Randolph AG, Cook DJ, Gonzales CA, Pribble CG. Ultrasound guidance for placement of central venous catheters: a metaanalysis of the literature. Crit Care Med 1996; 24: 2053-8.

10. Hind D, Calvert N, McWilliams $R$, et al. Ultrasonic locating devices for central venous cannulation: meta-analysis. BMJ 2003; 327: 361

11. Karakitsos D, Labropoulos N, De Groot E, et al. Real-time ultrasound-guided catheterisation of the internal jugular vein: a prospective comparison with the landmark technique in critical care patients. Crit Care 2006; 10: R162.

12. Shekelle $P G$, Dalas $P$. Use of real-time ultrasound guidance during central line insertion: brief update review. Making Health Care Safer II: An Updated Critical Analysis of the Evidence for Patient Safety Practices. Rockville, MD, USA: Agency for Healthcare Research and Quality; U.S. Department of Health and Human Services; 2013: 172-7.

13. Troianos CA, Hartman GS, Glas KE, et al. Guidelines for performing ultrasound guided vascular cannulation: recommendations of the American Society of Echocardiography and the Society of Cardiovascular Anesthesiologists. Anesth Analg 2012; 114: 46-72.

14. Shiver $S$, Blaivas $M$, Lyon $M$. A prospective comparison of ultrasound-guided and blindly placed radial arterial catheters. Acad Emerg Med 2006; 13: 1275-9.

15. Schwemmer U, Arzet HA, Trautner H, Rauch $S$, Roewer N, Greim $C A$. Ultrasound-guided arterial cannulation in infants improves success rate. Eur J Anaesthesiol 2006; 23: 476-80.

16. Tada T, Amagasa S, Horikawa $H$. Absence of efficacy of ultrasonic two-way Doppler flow detector in routine percutaneous arterial cannulation. J Anesth 2003; 17: 206-7.

17. Tada T, Amagasa S, Horikawa H. Usefulness of ultrasonic twoway Doppler flow detector in percutaneous arterial puncture in patients with hemorrhagic shock. J Anesth 2003; 17: 70-1.

18. Gu WJ, Tie HT, Liu JC, Zeng XT. Efficacy of ultrasound-guided radial artery catheterization: a systematic review and metaanalysis of randomized controlled trials. Crit Care 2014; 18: R93.

19. Tang $L$, Wang $F$, Li $Y$, et al. Ultrasound guidance for radial artery catheterization: an updated meta-analysis of randomized controlled trials. PloS One 2014; 9: e111527.

20. Bobbia $X$, Grandpierre $R G$, Claret $P G$, et al. Ultrasound guidance for radial arterial puncture: a randomized controlled trial. Am J Emerg Med 2013; 31: 810-5.

21. Levin $P D$, Sheinin $O$, Gozal $Y$. Use of ultrasound guidance in the insertion of radial artery catheters. Crit Care Med 2003; 31: 481-4. 
22. Tegtmeyer K, Brady $G$, Lai $S$, Hodo $R$, Braner D. Videos in clinical medicine. Placement of an arterial line. N Engl J Med 2006; 354: e13.

23. Hansen MA, Juhl-Olsen P, Thorn S, Frederiksen CA, Sloth E. Ultrasonography-guided radial artery catheterization is superior compared with the traditional palpation technique: a prospective, randomized, blinded, crossover study. Acta Anaesthesiol Scand 2014; 58: 446-52.

24. Ganesh A, Kaye R, Cahill AM, et al. Evaluation of ultrasoundguided radial artery cannulation in children. Pediatr Criti Care Med 2009; 10: 45-8.
25. Beaulieu $Y$, Laprise $R$, Drolet $P$, et al. Bedside ultrasound training using web-based e-learning and simulation early in the curriculum of residents. Crit Ultrasound J 2015; 7: 1-8.

26. Koh DB, Gowardman JR, Rickard CM, Robertson IK, Brown A. Prospective study of peripheral arterial catheter infection and comparison with concurrently sited central venous catheters. Crit Care Med 2008; 36: 397-402. 